\title{
Comparison of biophysical, biomechanical and ultrasonographic properties of skin in chronic dermatitis, psoriasis and lichen planus
}

\author{
Taraneh Yazdanparast ${ }^{1}{ }^{2}$, Kamran Yazdani ${ }^{3}$, Philippe Humbert ${ }^{4}$, Alireza Khatami ${ }^{1}$, Saman Ahmad Nasrollahi ${ }^{1}$, \\ Hournaz Hassanzadeh ${ }^{1}$, Amir Houshang Ehsani ${ }^{5}$, Leila Izadi Firouzabadi ${ }^{5}$, Alireza Firooz ${ }^{* 1,6}$
}

Received: 14 Nov 2017

Published: 5 Nov 2018

\section{Abstract}

Background: Skin biometrology is a useful method for evaluation of inflammatory skin disorders such as dermatitis, psoriasis, and lichen planus. The current study tries to compare the biophysical features of skin in dermatitis, psoriasis, and lichen planus.

Methods: By a convenient sampling method, 22 mild to moderate chronic dermatitis, 26 psoriasis, and 21 lichen planus patients were recruited in the study. Stratum corneum (S.C.) hydration, Transepidermal water loss (TEWL), pH, erythema, melanin, sebum, friction, elasticity parameters (R0, R2, and R5), skin temperature, skin thickness, and echo-density of epidermis and dermis were measured on the lesional (selected active lesion), uninvolved perilesional, and uninvolved symmetrical skin. The average of perilesional and symmetrical uninvolved parameters was used as control, while the percentage change of each parameter [(lesion control / control $\times 100$ ] was calculated, and compared among three diseases by ANOVA test using SPSS software version 18. The significance level was set at $\alpha=0.05$.

Results: Comparison of percentage changes showed that the changes in TEWL, friction index, sebum content, R2 (gross elasticity), R5 (net elasticity), skin temperature, dermal thickness, and epidermal density are not significantly different among three skin diseases. But there were significant differences in three diseases considering the decrease in S.C. hydration $(\mathrm{p}<0.001)$, R0 (opposed to firmness) $(\mathrm{p}<0.001)$, and dermal density $(\mathrm{p}<0.001)$ compared to control skin. Moreover, the increase in skin $\mathrm{pH}(\mathrm{p}<0.001)$, melanin content $(p=0.048)$, erythema $(p=0.023)$, and epidermal thickness $(p<0.001)$ significantly differed among these diseases.

Conclusion: Dermatitis, psoriasis and lichen planus lesions had specific biophysical changes. It may be helpful in their differential diagnosis.

Keywords: Biophysical properties, Dermatitis, Lichen planus, Psoriasis, Ultrasonographic properties

Conflicts of Interest: None declared

Funding: Grant from CRTSDL and two research awards provided by the Ministry of Health and Medical Education of Iran and Lierac Company

\section{*This work has been published under CC BY-NC-SA 1.0 license. \\ Copyright $\odot$ Iran University of Medical Sciences}

Cite this article as: Yazdanparast T, Yazdani K, Humbert Ph, Khatami A, Ahmad Nasrollahi S, Hassanzadeh H, Ehsani AH, Izadi Firouzabadi L, Firooz A. Comparison of biophysical, biomechanical and ultrasonographic properties of skin in chronic dermatitis, psoriasis and lichen planus. Med $J$ Islam Repub Iran. 2018 (5 Nov);32:108. https://doi.org/10.14196/mjiri.32.108

\section{Introduction}

Clinical examination is the most applicable tool of diagnosis in dermatology, while skin biopsy may be needed to confirm the diagnosis (1). Dermatitis, psoriasis, and lichen planus are all papulosquamous (PS) skin diseases with

Corresponding author:Dr Alireza Firooz, alifiruz@yahoo.com

1. Center for Research and Training in Skin Diseases and Leprosy, Tehran University of Medical Sciences, Tehran, Iran

2. Telemedicine Research Center, National Research Institute of Tuberculosis and Lung Diseases (NRITLD), Shahid Beheshti University of Medical Sciences, Tehran, Iran

3. Department of Epidemiology and Biostatistics, School of Public Health, Tehran University of Medical Sciences, Tehran, Iran

4. Centre d'Etudeset de Recherchesur le Tégument (CERT), INSERM UMR1098, SFR FED 4234 IBCT, University of Franche-Comté, Besançon, France

5. Department of Dermatology, Razi Hospital, Tehran University of Medical Sciences, Tehran, Iran

6. Clinical Trial Center, Tehran University of Medical Science, Tehran, Iran similar clinical pictures. Therefore a definitive histological diagnosis is necessary in many cases (2). Nowadays novel imaging techniques and non-invasive in vivo methods for measurement of skin biophysical properties are available

$\uparrow$ What is "already known" in this topic:

Skin biometrology is useful in evaluating inflammatory skin disorders such as dermatitis, psoriasis, and lichen planus.

$\rightarrow$ What this article adds:

Dermatitis, psoriasis and lichen planus lesions show specified biophysical changes that may help in differential diagnosis of them. 
to assess skin lesions. They can be used to increase diagnostic accuracy without any biopsy requirement $(1,3)$.

Skin biometrology is used to evaluate skin changes in some systemic diseases such as diabetes, thyroid dysfunction, and Ehlers-Danlos syndrome recently (4-6). There are also few studies about the skin biophysical changes in some papulosquamous diseases such as dermatitis (7) and psoriasis (8).

High-frequency ultrasound (HFUS) is another imaging technique which has been used to assess response to treatment in some papulosquamous conditions such as dermatitis, psoriasis and lichen planus (1). On the other hand, ultrasound features of skin in dermatitis, psoriasis, and lichen planus are different from each other $(9,10)$.

Overall, recent developments in imaging techniques with the ability to provide objective information, have made these instruments appropriate to be used for characterization of skin lesions with potential for clinical use in the future (1).

The aim of the study was to compare the biophysical, biomechanical and ultrasound features of skin in chronic dermatitis, psoriasis and lichen planus.

\section{Methods}

By a convenient sampling method, patients suffered from mild to moderate chronic dermatitis (atopic or contact dermatitis), psoriasis and lichen planus with a minimum age of 18 years referred to the Center for Research \& Training in Skin Diseases \& Leprosy (CRTSDL) from September 2014 to March 2016 who fulfilled eligibility criteria and provided informed consent were recruited in the study. The clinical diagnosis was made by a dermatologist and confirmed by histological findings. All participants had no treated active lesions with a duration of 2 to 4 weeks. The exclusion criteria included any systemic diseases that can affect skin conditions, recent history of any other skin diseases or operations in the previous three months, use of any systemic or topical medication or other interventions for their skin disease during the past two weeks, and pregnancy.

Baseline characteristics of the patients including age, gender, Fitzpatrick's skin type, location and duration of the disease were recorded.

To perform the biophysical assessments, participants were instructed not to wash or use any topical products on their skin from the night prior to the measurements. On the day of assessments, participants were asked to rest and relax for 20 minutes in a standard atmosphere condition (20-25 centigrade temperature; $25-30 \%$ humidity). Then the stratum corneum (S.C.) hydration (using Corneometer ${ }^{\circledR}$ CM 825), TEWL (using Tewameter ${ }^{\circledR}$ TM 300), pH (using Skin-pH-Meter ${ }^{\circledR} \mathrm{PH} 905$ ), erythema and melanin index (using Mexameter ${ }^{\circledR}$ MX 18), sebum (using Sebumeter ${ }^{\circledR}$ SM 815), friction value (using Frictiometer FR700), elasticity parameters including R0, R2, and R5 (using Cutometer ${ }^{\circledR} 580$ ), and skin temperature (using Skin-Thermometer ST 500) were measured by Multi Probe Adapter (MPA, Courage + Khazaka electronic $\mathrm{GmbH}$, Germany) on the lesional (selected active lesion), uninvolved perilesional, and uninvolved symmetrical skin.
Selection of these three sites was done by the dermatologist who had confirmed the diagnosis. The active border of a lesion was selected as lesional skin. Meanwhile, the normal-appearing skin of the same location on the opposite side of the body was selected as uninvolved symmetrical, and the normal-appearing skin, at least $3 \mathrm{~cm}$ away from the border of active disease, was selected as uninvolved perilesional skin.

It should be noted that frictiometer measures the torque as friction index and is related to elasticity and plasticity of skin. R0 (Uf) shows total elastic and plastic deformation of skin and the value of R0 is opposed to firmness. $\mathrm{R} 2$ shows gross elasticity and R2 is equal to $\mathrm{Ua} / \mathrm{Uf}$, where $\mathrm{Ua}$ is equal to viscoelastic/plastic recovery or final retraction of skin and Uf is equal to total deformation of skin. R5 shows net elasticity of the skin and R5 is equal to $\mathrm{Ur} / \mathrm{Ue}$, where $\mathrm{Ur}$ is equal to immediate elastic recovery or immediate retraction, and Ue is equal to immediate extensibility or elastic deformation (11).

High-frequency ultrasonography of the skin was done by $22 \mathrm{MHz}$ and $50 \mathrm{MHz}$ probes of DUB skin scanner (tpm Company, Luneburg, Germany) to measure the thickness and echo-density of dermis and epidermis, respectively, on the same lesional, perilesional, and symmetrical uninvolved skin.

As there was no clinically and statistically significant difference between perilesional and symmetrical uninvolved skin parameters, the means of these parameters were used as controls. The skin lesion parameters were compared with control by paired sample T- test.

In order to eliminate the impact of different anatomical site of measurements, the mean percentage for change of any of above-mentioned parameters was calculated according to the formula: [(lesion - control/control $) \times 100$, and compared among these three papulosquamous skin diseases.

The SPSS software version 18 (SPSS Inc., Chicago, US) was used for statistical analysis. Mean and standard deviation (SD) were used to describe quantitative data. Meanwhile, comparison of quantitative data among three diseases was performed by analysis of variance (ANOVA) test. Furthermore, if ANOVA showed significant differences, the post hoc comparisons (Tukey test) were done to compare data two by two. Statistical significance level was defined as $\mathrm{p}<0.05$.

The study was in accordance with the Declaration of Helsinki and approved by CRTSDL institutional review board and Tehran University of Medical Sciences ethics committee. Oral informed consents were provided by all the participants. All the measurements were non-invasive and done free of charge, and the data of the patients were kept confidential.

\section{Results}

Sixty-nine patients including 22 chronic dermatitis, 26 psoriasis, and 21 lichen planus patients were included in this study. The mean age of them was $40.10(\mathrm{SD}=14.37)$ years, and 37 were male. All patients had Fitzpatrick skin types III or IV. The mean duration of the disease was 5.47 $(\mathrm{SD}=7.81)$ years. The selected active lesions were located 
on upper limbs in 37 , lower limbs in 22 , trunk in 5 , and face in 5 patients.

In chronic dermatitis patients, stratum corneum hydration $(p<0.001)$, friction $(p=0.023)$, sebum $(p=0.016)$, and R0 $(p<0.001)$ were significantly lower in lesions, while TEWL $(\mathrm{p}=0.034), \mathrm{pH} \quad(\mathrm{p}=0.001)$, erythema $(\mathrm{p}=0.004)$, and temperature $(\mathrm{p}=0.049)$ were significantly higher. In psoriasis patients, stratum corneum hydration $(\mathrm{p}<0.001)$, friction $(\mathrm{p}=0.004)$, and $\mathrm{R} 0 \quad(\mathrm{p}<0.001)$ were significantly lower, while TEWL $(\mathrm{p}<0.001)$, $\mathrm{pH}$ $(\mathrm{p}<0.001)$, and melanin $(\mathrm{p}=0.008)$ were significantly higher in affected areas. In lichen planus patients, stratum corneum hydration $(\mathrm{p}<0.001)$, sebum $(\mathrm{p}=0.041)$, and $\mathrm{R} 0$ $(p<0.001)$ were significantly lower in lesions; on the other hand $\mathrm{pH}(\mathrm{p}<0.001)$, melanin content $(\mathrm{p}<0.001)$, erythema $(p<0.001)$, and temperature $(p=0.016)$ were significantly higher. Details of lesion-control comparisons for any diseases have been reported previously $(12,13)$.

Comparison of the percentage changes of the parameters among these three diseases by ANOVA test showed that the change in TEWL, friction index, sebum content, R2 (gross elasticity), R5 (net elasticity), skin temperature, dermal thickness, and epidermal density didn't show any statistically significant difference among skin diseases (Table 1).

But there were significant differences in three diseases considering the decrease in S.C. hydration, R0 (opposed to firmness), and dermal density compared to control skin (Table 1). Post hoc comparisons (Tukey test) showed that the decrease in lesion S.C. hydration in dermatitis $(p<0.001)$ and lichen planus $(p<0.001)$ were significantly less than psoriasis, and this decrease in lichen planus was significantly less than dermatitis $(\mathrm{p}<0.001)$, as well.

The decrease of lesion R0 in lichen planus was significantly less than psoriasis $(p<0.001)$, but the differences between dermatitis and psoriasis $(p=0.068)$ and between dermatitis and lichen planus $(p=0.070)$ were not significant.

The decrease of lesional dermal density in lichen planus was significantly less than dermatitis $(\mathrm{p}=0.026)$ and psoriasis $(p<0.001)$, but the difference between dermatitis and psoriasis was not significant $(\mathrm{p}=0.290)$.

On the other hand, of the increase in skin $\mathrm{pH}$, melanin content, erythema, and epidermal thickness significantly different among these diseases (Table 1). Post hoc comparisons (Tukey test) showed that the increase of lesion $\mathrm{pH}$ in dermatitis $(\mathrm{p}=0.001)$ and lichen planus $(\mathrm{p}=0.001)$ were significantly less than psoriasis, but the difference between dermatitis and lichen planus was not significant $(p=0.980)$. The increase of erythema in dermatitis was significantly less than lichen planus $(p=0.034)$, but the differences between dermatitis and psoriasis $(p=0.954)$ and between psoriasis and lichen planus $(\mathrm{p}=0.054)$ were not significant.

The increase of melanin content in dermatitis was significantly less than lichen planus $(\mathrm{p}=0.046)$, but the differences between dermatitis and psoriasis $(p=0.781)$ and between psoriasis and lichen planus $(p=0.157)$ were not significant.

The increases of lesional epidermal thickness in dermatitis $(p<0.001)$ and lichen planus $(p<0.001)$ were significantly less than psoriasis, but the difference between dermatitis and lichen planus was not significant $(\mathrm{p}=0.848)$.

\section{Discussion}

The results of this study showed that biometric changes of the lesions of chronic dermatitis, psoriasis and lichen planus vary among these papulosquamous diseases. Although the trends of decrease or increase of biophysical parameters were similar in all three groups, the percentage changes of them are different from each other and comparison of change percentages could be a way for differentiation.

Highest decrease of lesional S.C. hydration was formed in psoriasis, followed by dermatitis and lichen planus. Previous biometric studies have shown that S.C. hydration was lower on lesions in comparison with healthy skin in dermatitis and psoriasis $(14,15)$, but there is no such

Table 1. The comparison of skin biophysical parameters percentage change between dermatitis, psoriasis \& lichen planus and two by two comparisons by Tukey test

\begin{tabular}{|c|c|c|c|c|c|c|c|}
\hline \multirow[b]{2}{*}{ Variable } & \multicolumn{4}{|c|}{ Percentage change $($ mean $\pm \mathrm{SD} \dagger) \%$} & \multicolumn{3}{|c|}{ two by two (post hoc) comparisons } \\
\hline & Dermatitis & Psoriasis & Lichen planus & $\begin{array}{c}\text { ANOVA } \\
\text { (p) }\end{array}$ & $\begin{array}{c}\text { Dermatitis } \\
\text { \&Psoriasis } \\
\text { Post hoc } \\
\text { (p) }\end{array}$ & $\begin{array}{c}\text { Dermatitis } \\
\text { \&Lichen planus } \\
\text { Post hoc } \\
\text { (p) }\end{array}$ & $\begin{array}{c}\text { Psoriasis \& } \\
\text { Lichen planus } \\
\text { Post hoc } \\
\text { (p) }\end{array}$ \\
\hline Hydration & $-59.39 \pm 37.13$ & $-88.34 \pm 12.03$ & $-20.56 \pm 21.13$ & $<0.001$ & $<0.001$ & $<0.001$ & $<0.001$ \\
\hline TEWL & $149.16 \pm 259.45$ & $213.85 \pm 262.19$ & $104.28 \pm 138.08$ & 0.279 & - & - & - \\
\hline Friction & $-26.06 \pm 32.50$ & $-46.29 \pm 29.15$ & $-17.33 \pm 58.08$ & 0.059 & - & - & - \\
\hline $\mathrm{pH}$ & $7.61 \pm 9.10$ & $19.17 \pm 12.38$ & $6.99 \pm 9.85$ & $<0.001$ & 0.001 & 0.980 & 0.001 \\
\hline Sebum & $-67.56 \pm 49.51$ & $-28.30 \pm 85.48$ & $-6.17 \pm 182.69$ & 0.278 & - & - & - \\
\hline Melanin & $30.80 \pm 64.53$ & $46.44 \pm 70.25$ & $90.51 \pm 104.14$ & 0.048 & 0.781 & 0.046 & 0.157 \\
\hline Erythema & $27.00 \pm 36.06$ & $32.99 \pm 73.11$ & $82.03 \pm 91.55$ & 0.023 & 0.954 & 0.034 & 0.054 \\
\hline R0 & $-51.19 \pm 32.69$ & $-74.97 \pm 25.08$ & $-26.22 \pm 49.37$ & $<0.001$ & 0.068 & 0.070 & $<0.001$ \\
\hline $\mathrm{R} 2$ & $-3.91 \pm 38.80$ & $-46.79 \pm 111.66$ & $-9.34 \pm 26.25$ & 0.094 & - & - & - \\
\hline R5 & $27.72 \pm 96.21$ & $39.32 \pm 138.60$ & $-3.57 \pm 32.89$ & 0.354 & - & - & - \\
\hline Temperature & $1.93 \pm 4.00$ & $0.66 \pm 2.71$ & $1.32 \pm 2.37$ & 0.379 & - & - & - \\
\hline Dermal thickness & $16.93 \pm 32.36$ & $26.82 \pm 39.03$ & $15.17 \pm 28.53$ & 0.472 & - & - & - \\
\hline Dermal density & $-49.18 \pm 33.40$ & $-65.13 \pm 21.07$ & $-19.73 \pm 46.98$ & $<0.001$ & 0.290 & 0.026 & $<0.001$ \\
\hline Epidermal thickness & $15.53 \pm 19.89$ & $87.41 \pm 69.87$ & $6.55 \pm 17.95$ & $<0.001$ & $<0.001$ & 0.848 & $<0.001$ \\
\hline Epidermal density & $4.64 \pm 55.09$ & $-3.31 \pm 48.05$ & $-7.96 \pm 18.33$ & 0.714 & - & - & - \\
\hline
\end{tabular}


study in lichen planus. However, due to hyperkeratosis in lichen planus, decreased S.C. hydration is justifiable (16). The amount of S.C. hydration decrease varied among these three diseases, and this finding might be a point for distinction of them.

Elevated TEWL values are observed in a number of diseases with skin barrier abnormalities such as atopic dermatitis and psoriasis $(3,14)$. This finding was confirmed in this study, and there was no significant difference in the level of TEWL increase among these diseases, so TEWL can't be a way to differentiate them.

According to the previous assays, there is usually an inverse relationship between SC hydration and TEWL (3, 15). Although in recent studies the amount of TEWL increase didn't vary significantly among three diseases whereas S.C. hydration decrease did, the trend of SC hydration and TEWL changes was reciprocal as expected.

Superficial stiffness of lesion (shown by inverse R0) was highest in psoriasis compared with lichen planus and dermatitis, but the difference was only significant with lichen planus. It is known that psoriatic epidermis has the most keratinocyte hyperproliferation, as compared with dermatitis and lichen planus (17), and this could be the reason for increased stiffness. Moreover, higher stiffness could be due to a decrease of S.C. hydration (18) in psoriatic lesions, which had the least hydration among these diseases. The increase in skin stiffness has been demonstrated in psoriatic lesions previously with the decrease in R0 (19) and in another study with a different technique (CRRT: cutaneous resonance running time) (20).

Edema and cellular infiltration in inflammatory skin disorders were shown by a subepidermal low echogenic band (SLEB) and decrease in dermal echo-density (21, 22). We found that the decrease in lesional dermal density in lichen planus patients was lower than dermatitis and psoriasis. As it is shown that T-cell count in the dermis of lichen planus lesions was higher than psoriatic dermis (17), the contradictions may be due to different phase or severity of researched diseases. So, more studies in this regard are needed. Previous ultrasound studies have shown an intermediate zone between the epidermis and dermis in psoriasis (which its thickness correlated significantly with the PASI score) and a sound shadow in the dermis of lichen planus and chronic eczema (23).

It has been demonstrated that the increase in skin dryness and also TEWL in dermatitis patients, is accompanied by an increase in $\mathrm{pH}$ values (24). The present study revealed that psoriatic lesions which had the highest dryness and TEWL amongst three studied diseases showed the highest $\mathrm{pH}$.

Comparison of dermatitis, psoriasis and lichen planus by adaptive neuro-fuzzy inference systems had proved that the erythema in chronic dermatitis was less than psoriasis, and the melanin incontinence was a diagnostic feature for lichen planus (25). Our study also showed that the erythema and melanin content in lichen planus was significantly more than dermatitis and also more than psoriasis; though didn't reach statistical significance. Based on the similar findings of these two different assessment techniques, we may conclude that Mexameter assessment which shows the amount of erythema and melanin content might be helpful in the differentiation of these diseases.

The increase in thickness of epidermis in dermatitis and psoriasis due to hyperkeratosis and epidermal hyperproliferation has been shown histologically (17) and also by the non-invasive technique of optical coherence tomography (OCT) $(26,27)$. Comparison of epidermal thickness in dermatitis, psoriasis, and lichen planus histology revealed that psoriasis had the most well-marked epidermal hyperproliferation and atopic dermatitis and lichen planus had less epidermal hyperproliferation, respectively (17). The same comparison by confocal microscopy also showed that thickened epidermis is the main significant criterion for psoriasiform dermatitis and mild thickening of the epidermis was detected in a few cases of sub-acute eczema (28). The epidermal thickness measured by ultrasonography was significantly more in psoriasis than dermatitis and lichen planus in this study. Thickening of epidermis in dermatitis and psoriasis plaques was shown by ultrasonography previously $(22,29)$ but this is the first study comparing them.

A limitation of the study is that the duration and severity of the diseases may possibly influence the measurements and make difficult to compare them. We included only patients with mild to moderate diseases (according to dermatologist diagnosis) to deal with this limitation.

The strength of the study is that it is the first comprehensive comparative evaluation of biophysical, biomechanical and ultrasonographic findings of dermatitis, psoriasis and lichen planus.

Histopathology is still considered as a gold standard tool to differentiate papulosquamous skin disorders $(2,30)$ but recently non-invasive methods like confocal laser scanning microscopy (CLSM) has been used for the diagnosis of inflammatory diseases such as psoriasis, lichen planus and lupus erythematosus (31).

\section{Conclusion}

According to the results of this study, skin biometrology can be considered as a non-invasive and valuable technique to help in the diagnosis of papulosquamous skin disorders such as dermatitis, psoriasis and lichen planus. These new technologies allow dermatologists to do a fast, objective and quantitative assessment of skin to make the diagnosis and evaluate the response to treatment.

\section{Acknowledgments}

This study was supported by a research grant from CRTSDL and two research awards provided by the Ministry of Health and Medical Education of Iran and Lierac Company.

\section{Conflict of Interests}

The authors declare that they have no competing interests.

\section{References}

1. Dasgeb B, Kainerstorfer J, Mehregan D, Van Vreede A, Gandjbakhche A. An introduction to primary skin imaging. Int $\mathrm{J}$ Dermatol. 2013;52(11):1319-30. 
2. Hosamane S, Pai M, Philipose TR, Nayarmoole U. Clinicopathological Study of Non-Infectious Erythaematous Papulosquamous Skin Diseases. J Clin Diagn Res. 2016;10(6): EC19EC22.

3. Darlenski R, Sassning S, Tsankov N, Fluhr J. Non-invasive in vivo methods for investigation of the skin barrier physical properties. Eur J Pharm Biopharm. 2009;72(2):295-303.

4. Szepetiuk G, Piérard G, Betea D, Petrossians P, Xhauflaire-Uhoda E, Beckers A, et al. Biometrology of physical properties of skin in thyroid dysfunction. J Eur Acad Dermatol Venereol. 2008;22(10):1173-7.

5. Catala-Pétavy C, Machet L, Georgesco G, Pétavy F, Maruani A, Vaillant L. Contribution of skin biometrology to the diagnosis of the Ehlers-Danlos syndrome in a prospective series of 41 patients. Skin Res Technol. 2009;15(4):412-7.

6. Seirafi H, Farsinejad K, Firooz A, Davoudi S, Robati R, Hoseini M, et al. Biophysical characteristics of skin in diabetes: a controlled study. J Eur Acad Dermatol Venereol. 2009;23(2):146-9.

7. Firooz A, Gorouhi F, Davari P, Atarod M, Hekmat S, RashighiFiroozabadi M, et al. Comparison of hydration, sebum and $\mathrm{pH}$ values in clinically normal skin of patients with atopic dermatitis and healthy controls. Clin Exp Dermatol. 2007;32(3):321-2.

8. Cannavò SP, Guarneri F, Giuffrida R, Aragona E, Guarneri C. Evaluation of cutaneous surface parameters in psoriatic patients. Skin Res Technol. 2017 Feb;23(1):41-47.

9. El-Zawahry M, Abdel E-HE-CH, Abd-El-Rahman RS, Ahmed BD, Mohamed FM. Ultrasound biomicroscopy in the diagnosis of skin diseases. Eur J Dermatol. 2007;17(6):469-75.

10. Dańczak-Pazdrowska A, Polańska A, Silny W, Sadowska A, Osmola-Mańkowska A, Czarnecka-Operacz M, et al. Seemingly healthy skin in atopic dermatitis: observations with the use of highfrequency ultrasonography, preliminary study. Skin Res Technol. 2012;18(2):162-7.

11. Neto P, Ferreira M, Bahia F, Costa P. Improvement of the methods for skin mechanical properties evaluation through correlation between different techniques and factor analysis. Skin Res Technol. 2013;19(4):405-16.

12. Yazdanparast T, Yazdani K, Humbert P, Khatami A, Ahmad Nasrollahi S, Firooz A. Biophysical properties of human skin in subacute and chronic eczema: comparison of involved and uninvolved areas, Proceedings of the 25th EADV (European academy of dermatology and venereology) congress; 2016: September 28-October 2; Vienna, Austria.

13. Yazdanparast T, Hasanzadeh H, Ahmad Nasrollahi S, Khatami A, Yazdani K, Humbert $\mathrm{P}$, et al. Biophysical and biomechanical properties of psoriasis lesions in comparison with unaffected skin, Proceedings of the 25th EADV (European academy of dermatology and venereology) congress; 2016: September 28 - October 2; Vienna, Austria.

14. De Marchi F, Piacentini GL, Piazza M, Sandri M, Boner AL, Peroni DG. Correlation of skin barrier impairment in atopic dermatitis with aeroallergen sensitization. Allergy Asthma Proc. 2015;36(6):e127-33.

15. Lee Y, Je YJ, Lee SS, Li ZJ, Choi DK, Kwon YB, et al. Changes in transepidermal water loss and skin hydration according to expression of aquaporin-3 in psoriasis. Ann Dermatol. 2012; 24(2):168-74.

16. Wagner G, Rose C, Sachse M. Clinical variants of lichen planus. J Dtsch Dermatol Ges. 2013;11(4):309-19.

17. Bovenschen H, Seyger M, Van De Kerkhof P. Plaque psoriasis vs. atopic dermatitis and lichen planus: a comparison for lesional T-cell subsets, epidermal proliferation and differentiation. Br J Dermatol. $2005 \mathrm{Jul} ; 153(1): 72-8$.

18. Del Rosso JQ, Levin J. The clinical relevance of maintaining the functional integrity of the stratum corneum in both healthy and disease-affected skin. J Clin Aesthet Dermatol. 2011 Sep;4(9):22-42.

19. Choi JW, Kwon SH, Youn JI, Youn SW. Objective measurements of erythema, elasticity and scale could overcome the inter-and intraobserver variations of subjective evaluations for psoriasis severity. Eur J Dermatol. 2013;23(2):224-9.

20. Lv C, Song S, Luo W, Elias PM, Man MQ. Cutaneous resonance running time is decreased in psoriatic lesions. Skin Res Technol. 2012;18(2):232-7.

21. Polańska A, Dańczak-Pazdrowska A, Silny W, Jenerowicz D, OlekHrab K, Osmola-Mańkowska A. Nonlesional skin in atopic dermatitis is seemingly healthy skin-observations using noninvasive methods. Wideochir Inne Tech Maloinwazyjne. 2013;8(3):192-199.
22.-Polańska A, Dańczak-Pazdrowska A, Silny W, Woźniak A, Maksin $\mathrm{K}$, Jenerowicz $\mathrm{D}$, et al. Comparison between high-frequency ultrasonography (Dermascan C, version 3) and histopathology in atopic dermatitis. Skin Res Technol. 2013;19(4):432-7.

23. El-Zawahry MB, Abdel El-Hameed El-Cheweikh HM, Abd-ElRahman Ramadan S, Ahmed Bassiouny D, Mohamed Fawzy M. Ultrasound biomicroscopy in the diagnosis of skin diseases. Eur J Dermatol. 2007;17(6):469-75.

24. Jungersted JM, Scheer H, Mempel M, Baurecht H, Cifuentes L, Høgh JK, Hellgren LI, Jemec GB, Agner T, Weidinger S. Stratum corneum lipids, skin barrier function and filaggrin mutations in patients with atopic eczema. Allergy. $2010 \mathrm{Jul} ; 65(7): 911-8$.

25. Übeylı ED, Güler I. Automatic detection of erythemato-squamous diseases using adaptive neuro-fuzzy inference systems. Comput Biol Med. 2005;35(5):421-33.

26. Gambichler T, Jaedicke V, Terras S. Optical coherence tomography in dermatology: technical and clinical aspects. Arch Dermatol Res. 2011;303(7):457-73.

27. Morsy H, Kamp S, Thrane L, Behrendt N, Saunder B, Zayan H, et al. Optical coherence tomography imaging of psoriasis vulgaris: correlation with histology and disease severity. Arch Dermatol Res. 2010;302(2):105-11.

28. Ardigo M, Longo C, Gonzalez S. Multicentre study on inflammatory skin diseases from The International Confocal Working Group: specific confocal microscopy features and an algorithmic method of diagnosis. Br J Dermatol. 2016 Aug;175(2):364-74.

29. Gutierrez M, Wortsman X, Filippucci E, De Angelis R, Filosa G, Grassi W. High-frequency sonography in the evaluation of psoriasis: nail and skin involvement. J Ultrasound Med. 2009;28(11):1569-74.

30. Abdel-Azim NE, Ismail SA, Fathy E. Differentiation of pityriasis rubra pilaris from plaque psoriasis by dermoscopy. Arch Dermatol Res. 2017 May; 309(4):311-314.

31. Sattler E, Maier T, Hoffmann V, Hegyi J, Ruzicka T, Berking C. Noninvasive in vivo detection and quantification of Demodex mites by confocal laser scanning microscopy. $\mathrm{Br} \quad \mathrm{J}$ Dermatol. 2012;167(5):1042-7. 